\title{
Some evidence that fluoride during orthodontic treatment reduces occurrence and severity of white spot lesions
}

\author{
Is fluoride effective in preventing white spot lesions during orthodontic \\ treatment?
}

\begin{abstract}
Benson PE, Parkin N, Millett DT, Dyer FE, Vine S, Shah A. Fluorides for the prevention of white spots on teeth during fixed brace treatment (Cochrane Review). In the Cochrane Library. Chichester: John Wiley; 2004, Issue 3
\end{abstract}

Data sources Sources were the Cochrane Oral Health Group's Trials Register, the Cochrane Central Register of Controlled Trials (CENTRAL), Medline and Embase. The authors of identified trials were contacted for further data.

Study selection Studies were selected if they were randomised clinical trials (RCT) or quasi-RCT, involved the use of a fluoridecontaining product compared with no use or use of a non-fluoride control, and if enamel demineralisation was assessed during or after orthodontic treatment.

Data extraction and synthesis Six reviewers independently, in duplicate, extracted data. The primary outcome was the difference in the presence or absence of white spots between experimental and control patients for parallel design studies, and between experimental and control quadrants, for split-mouth design studies. Potential sources of heterogeneity were examined. Sensitivity analyses were undertaken for the items assessed for quality and publication bias.

Results A total of 15 trials, with 723 participants, provided data for this review. None of the studies fulfilled all of the methodological quality assessment criteria. There is some evidence, however, that a daily sodium fluoride mouthrinse reduces the severity of enamel decay surrounding a fixed brace [weighted mean difference for lesion depth, $-70.0 \mu \mathrm{m} ; 95 \%$ confidence interval $(\mathrm{Cl}),-118.2$ to $-21.8 \mu \mathrm{m}]$. Use of a glass ionomer cement for bracket bonding reduces the prevalence (Peto odds ratio, $0.35 ; 95 \% \mathrm{Cl}, 0.15-0.84$ ) and severity of white spots (weighted mean difference for mineral loss, -645 vol\% $\mu \mathrm{m} ; 95 \% \mathrm{Cl}$, -915 to -375 ) compared with composite resins.

Conclusions There is some evidence that the use of topical fluoride or fluoride-containing bonding materials during orthodontic treatment reduces the occurrence and severity of white spot lesions, but there is little evidence regarding which method or combination of methods is most effective for fluoride delivery. Based on current best practice in other areas of dentistry for which there is evidence, we recommend that patients with fixed braces rinse daily with a $0.05 \%$ sodium fluoride mouthrinse. More high-quality, clinical research is required into the different modes of delivering fluoride to the orthodontic patient.
Address for correspondence: Emma Tavender, Review Group Co-ordinator, Cochrane Oral Health Group, University Dental Hospital of Manchester, Higher Cambridge Street, Manchester M156FH, UK. E-mail: emma.tavender@man.ac.uk

\section{Commentary}

The review addresses two issues that are contemporary and largely unanswered by current available evidence. White spot lesions continue to be a real issue with fixed orthodontic appliance therapy and, although fluorides have been the subject of reviews over previous years, none of the studies have looked specifically at use of the various topical fluoride delivery systems in children or adolescents undergoing fixed orthodontic therapy.

Which is the best vehicle for local delivery of fluorides? The perspectives are well-defined for this review and the reviewers did well to focus two specific types of fluorides, that is, topical applications or rinses and the fluoride-release cements, elastics or compomers.

The outcome considered for this topic needs to be a reduction in the occurrence and severity of white spots. The occurrence is easier to determine than the severity, which would warrant a study into the depth of the lesion or extent of demineralisation - not be possible unless the teeth were extracted. The use of randomised or quasi-randomised designs with an intervention and appropriate control, in terms of a fluoride and non-fluoride product, is simple and direct and in agreement with the basic research questions raised by the reviewers.

The reviewers have been very comprehensive and thorough in the inclusion of relevant studies, due regard having been given to stringent methodological filters and quality checks. It is a wonder that even those 15 studies that finally provided data for the review survived the parameters laid down. The inherent issues of generating good-quality evidence are brought into sharp focus when one notes the various issues involved. In terms of randomisation and allocation concealment, there is a wide gap between different groups of studies. Only six trials report a blinding of outcomes and if one was to go by the minor methodological quality checks prescribed by the reviewers, only one study survives.

The strength of the review emerges from the detailed comparison and consideration of each method of delivery of fluorides. Care was taken to assess each method and the fact that wherever possible the original authors were approached to confirm their method only adds to the validity of the review. The variability between various studies requires a constructive analysis. Stringency in applying established principles of review would lead to a loss of valuable inferences, and this is where the authors have scored. In spite of the variability in methodology, assessment criteria and other parameters, they were able to construct a valuable insight into both the clinical and research issues. Local delivery of fluorides will reduce white spot lesions in an orthodontic patient undergoing fixed banded therapy. There is no dispute on this issue at all. It is the most effective method of delivery that is still debatable. Fluoride mouthrinses do act in a beneficial manner but rely largely on patient compliance, which in adolescents is an issue in itself. A fluoride cement or elastomer is definitely a better method of delivery because these release fluoride into the micro-environment 
around the bracket, where it is actually required, and are not dependent upon patient compliance. The issue with these methods of delivery is the fact that, after an initial jump in fluoride levels, there is a rapid dissipation. Therefore, is the concentration of fluorides adequate to prevent the development of the lesion?

The evidence on the beneficial fluoride delivery of glass ionomer cements is weak, whereas elastomerics work but there are issues with costs and availability. One cannot but help agree with the clinical bottom line of the review that the best practice would be to recommend a $0.05 \%$ sodium fluoride mouthrinse daily. If other methods of local fluoride delivery can be added, so be it! This review has universal appeal and only strengthens the cause of an evidence-based quest for knowledge from information. Apart from a clear clinical bottom line, the future trends in research emerge rather clearly. Future directions lie in devising the best method of delivery of fluorides, the apparent benefit being an amply established reputation for the reviewers!

\section{Practice point}

- Local fluoride delivery during orthodontic treatment reduces white spot lesions, but more evidence is needed on the best delivery method.

\section{Anmol Kalha}

Department of Orthodontics and Dentofacial Orthopaedics, College of Dental Sciences, Davangere, Karnataka, India

Evidence-Based Dentistry (2004) 5, 98-99.

doi:10.1038/sj.ebd.6400298 